\title{
ENSAYOS DE REFORESTACION POR SIEMBRA DIRECTA CON ALGARROBILLA (Balsamocarpon brevifolium CLOS) EN LA ZONA DE VALLENAR
}

Johannes Wrann H. $\left(^{*}\right)$

Daniel Barros R. (*)

\section{RESUMEN}

Algarrobilla (Balsamocarpon brevifolium CLOS) es un arbusto nativo de la zona árida (III y IV Región) de Chile. Dado su gran atractivo como productora de leña y frutos con un alto contenido de taninos, la especie ha sido sobreexplotada.

Basado en una experiencia previa de INFOR, que también se analiza en este artículo, se instaló un ensayo de siembra directa en la zona costera de la provincia de Huasco ( $28^{\circ} 36^{\prime \prime}$ Lat.S.) en invierno de 1986 (Junio). El lugar tiene características severas de aridez, llegando su precipitación anual hasta $100 \mathrm{~mm}$. Los suelos son delgados y de desarrollo incipiente.

Se obtuvieron resultados promisorios para la repoblación con esta especie, recomendándose un riego mensual ( 8 lt por casilla de $50 \mathrm{~cm} \times 50 \mathrm{~cm}$ ), incluyendo "mulch" (capa de paja de 3 cm de espesor sobre el suelo), escarificación del suelo hasta 20 $\mathrm{cm}$ de profundidad y control de roedores.

\section{ABSTRACT}

"Algarrobilla" (Balsamocarpon brevifolium CLOS) is a native shrub from Chile with a limited occurrence in the arid region of this country. The species is interesting due to the fuelwood and high tannin content in the fruit, and was therefore overexploited en the past.

Based on a previous experience also carried out by INFOR (and analized in this article too), a direct seeding trial was established near the coast in the Huasco province $\left(28^{\circ} 36^{\prime \prime} \mathrm{S}\right.$. Lat.) during the winter 1986 (June). After the first dry season, in autumn 1987, the evaluation was made, concluding that the best treatment is an intensive soil preparation (scarification down to $20 \mathrm{~cm}$ ), mulching ( $3 \mathrm{~cm}$ thick straw layer over the soil surface), a monthly irrigation ( 8 lt per $50 \mathrm{~cm} \times 50 \mathrm{~cm}$ seed bed) and a rodent control.

(*) Ingenieros Forestales, División Silvicultura. Instituto Forestal. Huérfanos 554. Santiago - Chile. 


\section{INTRODUCCION}

"Algarrobilla" (Balsamocarpon brevifolium CLOS) es un arbusto leguminoso (familia Caesalpinaceae $)$ nativo de la zona árida del norte de Chile $\left(28^{\circ}-29^{\circ} 50^{\circ}\right.$ Lat.S.). Este arbusto alcanza una altura de 1 a $3 \mathrm{~m}$. Debido a su extraordinaria adaptación a un medio árido riguroso (precipitación anual hasta $100 \mathrm{~mm}$ ) y su alto contenido de tanino de buena calidad en su fruto $(54-78 \%)$, esta especie debería ser considerada como una alternativa interesante en programas de forestación para estas áreas.

La madera de este arbusto es apreciada como combustible, razón por la cual ha sido sometido a una explotación intensa en el pasado (ORTIZ, G., 1943; ESCOBAR, A., 1946).

La regeneración natural de la Algarrobilla es extremadamente difícil, debido a las condiciones climáticas y a los suelos delgados de formación incipiente donde crece. De acuerdo a observaciones a través de varios períodos, la producción de semillas de Algarrobilla depende de la cantidad de lluvias caídas durante el invierno anterior a la fructificación (ORTIZ, G., 1943). Por otra parte, las ratas se alimentan de las semillas (HOWES, F.N., 1953), y el ganado caprino puede significar un serio peligro para el establecimiento de la regeneración.

Las referencias sobre intentos de forestación en el pasado (ORTIZ, G., 1943) son escasas; en consecuencia, se conoce muy poco acerca de las posibilidades de repoblamiento y la tasa de crecimiento de la especie.

La producción de plantas es seriamente afectada por hongos (DONOSO, J., 1985) y se ha investigado el control apropiado (MONTENEGRO, G., y ARCE, P., 1987).

\section{OBJETIVO}

El objetivo de este estudio es lograr un método de forestación apropiado con Algarrobilla en su área de distribución natural en el norte de Chile, III Región.

\section{ANTECEDENTES DE LAS AREAS DE ENSAYO}

\section{Ensayos establecidos en 1985}

En 1985 se establecieron dos ensayos dentro del área natural de distribución de la especie, considerando para esto un sector costero y de precordillera (Figura 1):

a) Un ensayo en el área costera, al oeste de la ciudad de Vallenar, cerca del pueblo de Freirina. El lugar se llama "Totora", $28^{\circ} 36^{\prime}$ Lat.S.; 71 ${ }^{\circ}-06^{\prime}$ Long.W; $625 \mathrm{~m}$ de Altitud. La exposición es Norte con una pendiente de alrededor de $40 \%$.

b) Un ensayo al interior en la precordilllera de los Andes, al N.E. de Vallenar, $28^{\circ} 17$ Lat.S.; $72^{\circ} 23^{\prime}$ Long.W.; $920 \mathrm{~m}$ Altitud. La exposición es W. y la pendiente de alrededor del $15 \%$.

\section{Ensayos establecidos en 1986}

Se estableció en el mismo lugar costero descrito en el punto anterior (a).

\section{Clima}

De acuerdo a la clasificación de Koeppen, se encuentra el Clima de Desierto con Nubosidad Abundante (BW) en la zona costera y Clima de Desierto Marginal bajo (BWh) en la zona de precordillera (FUENZALIDA, H., 1967). De acuerdo a la clasificación de Emberger (DI CASTRI y HAJEK, 1976) el clima es Mediterráneo Arido en ambas áreas.

Algunos datos climáticos se muestran en el cuadro I (ANTONIOLETTI et al; ALMEY. DA, 1958; DI CASTRI y HAJEK, 1976; SANTIBAÑEZ, 1973, cit. en CORFO, 1984). 
De estos datos se puede observar que el clima es árido y de condiciones extremadamente secas para el establecimiento de especies arbóreas o arbustivas.

Suelo

De acuerdo a los Grandes Grupos de Suelos (ROBERTS y DIAZ, 1959 - 60) los suelos presentes en el área corresponden a los Suelos Rojos de Desierto. Estos son muy delgados, de

\section{CUADRO 1}

DATOS CLIMATICOS PROMEDIOS DEL AREA DE ESTUDIO

\begin{tabular}{|c|c|c|c|}
\hline Variable & Costa & Interior & $\begin{array}{c}\text { Precordillera } \\
\text { Andina }\end{array}$ \\
\hline $\begin{array}{l}\text { Temperatura media anual }\left({ }^{\circ} \mathrm{C}\right) \\
\text { Temperatura máx. media anual }\left({ }^{\circ} \mathrm{C}\right) \\
\text { Temperatura mín. media anual }\left({ }^{\circ} \mathrm{C}\right) \\
\text { Precipitación (mm/año) } \\
\text { Días despejados por año } \\
\text { Humedad relativa anual }(\%) \\
\mathrm{N}^{\circ} \text { de meses secos por año } \\
\mathrm{N}^{\circ} \text { Días libres heladas/año }\end{array}$ & $\begin{aligned} 17 \\
22-25 \\
8-9 \\
57-100 \\
44 \\
66-78 \\
11-12 \\
365-350\end{aligned}$ & $\begin{array}{rr}15 & \\
25- & 30 \\
3- & 8 \\
58 & \\
190 & \\
50- & 60 \\
11- & 12 \\
350-180\end{array}$ & $\begin{array}{cr}11 \\
18-25 \\
0-3 \\
100 \\
264 \\
28 \\
11 \\
180-150\end{array}$ \\
\hline
\end{tabular}

\section{FIGURA 1}

\section{UBICACION ENSAYOS}

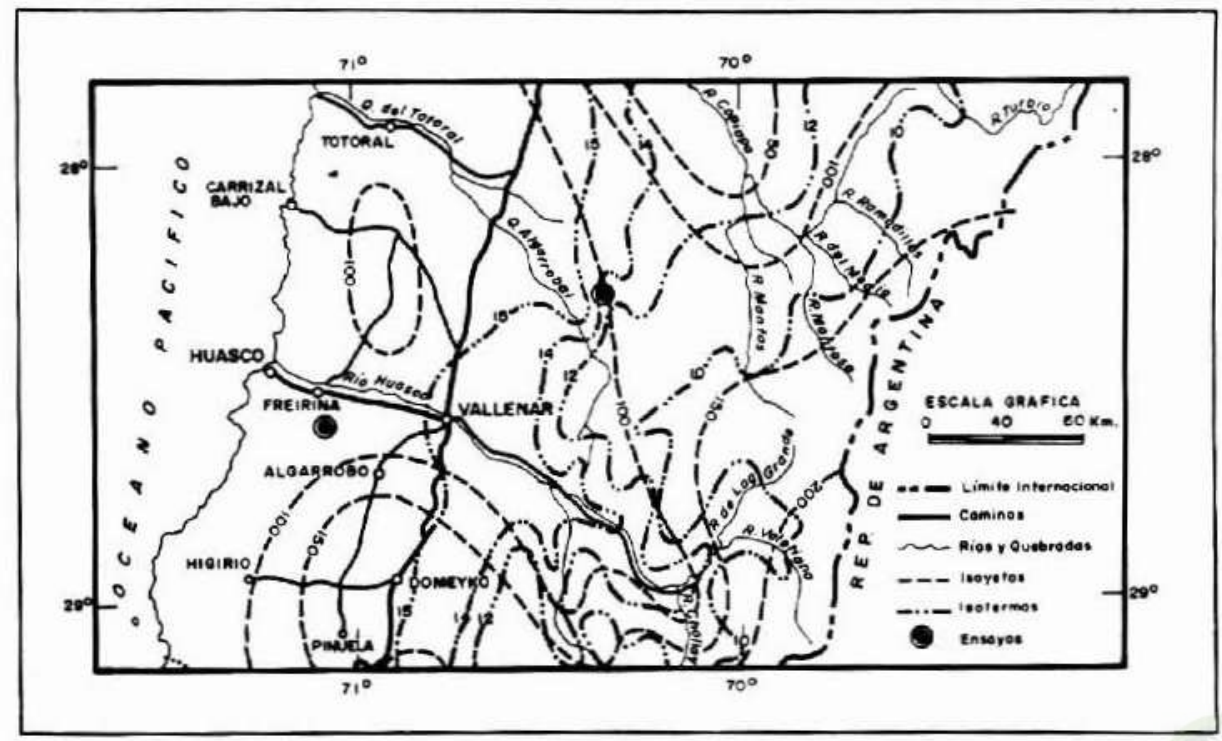


textura gruesa, arenosos, de estructura débil y en un estado de desarrollo incipiente. El análisis de los elementos solubles muestra que los cationes principales son $\mathrm{Ca}$ y $\mathrm{Na}$ (CORFO, 1984).

En ambos lugares de ensayo se tomó una muestra superficial del suelo (hasta $20 \mathrm{~cm}$ de profundidad). Se efectuó un análisis de textura en el laboratorio de INFOR; el análisis de fertilidad, salinidad y capacidad de retención de agua se efectuó en el Laboratorio de la Universidad Católica de Chile, en Santiago (Cuadro 2).

Los antecedentes indican que el suelo es arenoso, sin limitantes de fertilidad, con muy baja cantidad de materia orgánica. Son suelos básicos pero no salinos. Debido a su textura tienen una baja capacidad de retención de agua.

\section{CUADRO 2}

ANALISIS DEL SUELO SUPERFICIAL

\begin{tabular}{|c|c|c|}
\hline \multirow[t]{2}{*}{ Variable } & \multicolumn{2}{|c|}{ Lugar de Ensayo } \\
\hline & La Totora (costa) & La Jarilla (interior) \\
\hline $\begin{array}{l}\text { a) Textura } \\
\text { Arena-arcilla-limo (\%) } \\
\text { b) Fertilidad } \\
\mathrm{N}-\mathrm{P}-\mathrm{K}(\mathrm{ppm}) \\
\text { Materia orgánica (\%) } \\
\text { c) Salinidad (mmhos/cm) } \\
\text { d) } \mathrm{pH} \\
\text { e) Capacidad retención agua } \\
0,3 \text { (bar) } \\
15 \text { (bar) }\end{array}$ & $\begin{array}{c}90-3,3-67 \\
26-12,0-227 \\
0,37 \\
0,14 \\
8,06 \\
\\
7,68 \% \\
4,33 \%\end{array}$ & $\begin{array}{c}50-16,7-33,3 \\
33-24,0-386,0 \\
0,74 \\
0,22 \\
8,08 \\
11,67 \% \\
6,90 \%\end{array}$ \\
\hline
\end{tabular}

\section{METODOLOGIA}

Las áreas de ensayo se cercaron en una extensión de $60 \mathrm{~m}$ x $40 \mathrm{~m}$ con alambre de malla de $3 / 4$ pulgadas de abertura de tejido.

Se instalaron ensayos en los años 1985 y 1986, los que se describen a continuación:

\section{Ensayos establecidos en 1985}

Cada una de las parcelas fue subdividida en 6 bloques de tamaño y forma irregular, con el objeto de contar con ejemplares adultos que sirvieran de sombra para algunos tratamientos. Dentro de cada bloque se establecieron los tratamientos, cada uno en una unidad de muestreo (subparcela) de cuatro casillas de $50 \mathrm{~cm} \times 50 \mathrm{~cm}$ para la siembra. Los tratamientos fueron los siguientes:
a) Escarificación del suelo - sombra - riego
b) Escarificación del suelo - mulch - sombra - riego
c) Escarificación del suelo - riego
d) Escarificación del suelo
e) Testigo 


\section{J. WRANN H. y D. BARROS R.}

La escarificación del suelo se efectuó hasta una profundidad de $20 \mathrm{~cm}$ y el suelo se harneó con una malla de $2 \mathrm{~cm}$. de abertura. Las casillas quedaron $5 \mathrm{~cm}$ bajo la superficie.

El tratamiento de sombra significa que las casillas se ubicaron bajo la copa de un ejemplar adulto de Algarrobilla presente en el bloque. De esta manera el grado de sombra entre las casillas fue distinto, dependiendo de la orientación o densidad de las ramas del arbusto que proporciona la sombra. El riego consistió en la aplicación de 7,5 - 8 lt de agua por casilla, inmediatamente antes de la siembra y luego cinco veces por año, distribuido desde el invierno de 1985 al otoño de 1986. El agua se aplicó lentamente, evitando provocar escurrimiento.

El mulch consistió en una mezcla de suelo con paja de trigo de alrededor de $2 \mathrm{~cm}$ de profundidad en la casilla y una capa de paja de $2-3 \mathrm{~cm}$ de espesor puesto sobre la superficie de la casilla.

Las semillas a sembrar fueron analizadas previamente a objeto de asegurar una germinación del $75-80 \%$.

\section{FIGURA 2}

PARCELA DEL ENSAYO COSTERO (40 m x $60 \mathrm{~m}$ aprox.)

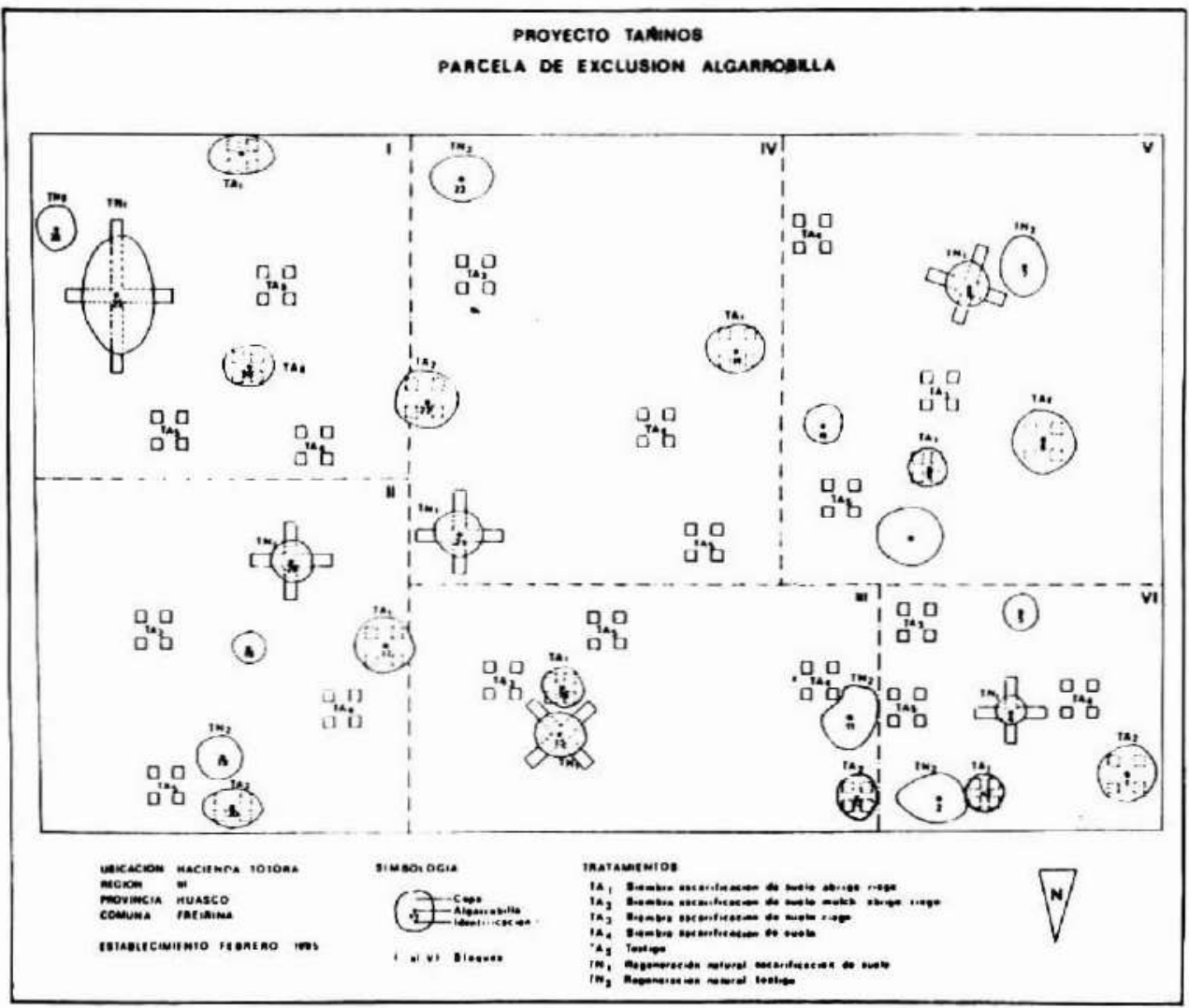


Las semillas a usar en los tratamientos con riego fueron sumergidas durante 60 minutos en ácido sulfúrico técnico concentrado y durante 10 minutos aquellas semillas a usar en los tratamientos sin riego. De esta manera las semillas del tratamiento sin riego pueden mantener su latencia por más tiempo. Después de esto las semillas fueron lavadas en agua corriente y desinfectadas con un insecticida - fungicida (Pomarsol H - Bayer).

El número de semillas sembradas por casilla fue 25 .

La siembra se efectuó en Abril (otoño) de 1985 y se repitió en Junio del mismo año, debido a que la germinación había sido insuficiente y se observaron daños por herbívoros.

Dentro del año se aplicó dos veces un repelente contra conejos (Pomarsol F - Bayer - i.a. Thiuram $80 \%$ ) en forma de lechada alrededor de las casillas con una concentración de $1 \mathrm{~kg}$ Pomarsol por 8 lt de agua.

\section{Ensayo establecido en 1986}

Dentro de la misma parcela cercada del lugar costero (La Totora) se estableció un nuevo experimento en Junio 1986, basado en la experiencia obtenida durante el año anterior. La parcela grande cercada se dividió en cinco bloques, obteniéndose un diseño de bloques con 5 repeticiones. Se usó la misma unidad muestreal (4 casillas).

Las semillas también fueron analizadas previamente y tratadas con ácido sulfúrico a objeto de asegurar la germinación, pero esta vez se sumergieron durante 90 minutos. Ensayos de laboratorio indicaron una mejor germinación con este tiempo de tratamiento.

Se usaron los siguientes tratamientos, que indican dos intensidades de preparación de suelo y tres frecuencias de riego:
a) $I F_{1}$
e) $\mathrm{MF}_{1}$
b) $I F_{2}$
f) $\mathrm{M} \mathrm{F}_{2}$
c) ${\mathrm{I} \mathrm{F}_{3}}_{3}$
g) $\mathrm{MF}_{3}$
d) $\mathrm{I} \mathrm{H}$
h) $\mathrm{M} \mathrm{H}$

Donde:

I = preparación intensiva de suelo, igual que en 1985, pero sin harnear, extrayendo las piedras más grandes

$\mathrm{M}$ = preparación moderada de suelo, escarificación hasta una profundidad aproximada de $5 \mathrm{~cm}$.

$F_{1} \quad=$ riego una vez cada 3 meses

$\mathrm{F}_{2} \quad$ = riego una vez cada 2 meses

$\mathrm{F}_{3} \quad$ = riego una vez cada 1 mes

$\mathrm{H}$ = incorporación de Hydro-Growth (nombre comercial), un polímero absorbente de agua mezclado con el suelo $10 \mathrm{gr}$ por casilla de siembra. Se usó frecuencia de riego cada 3 meses.

La cantidad de agua aplicada por riego fue la misma de 1985. Cada tratamiento recibió un riego junto con la siembra (Junio 1986) y luego en Agosto 1986. Las frecuencias de riego fueron aplicadas desde Octubre 1986 hasta Marzo de 1987.

Con el objeto de controlar las ratas se efectuó antes de la siembra una aplicación de cebos venenosos usando un raticida doméstico común (Racumin - Bayer) mezclado con $1 \mathrm{Kg}$ de avena, distribuidos en diez puntos dentro de la parcela experimental grande. 
En cada tratamiento se aplicó el mulch como el tratamiento "b" del ensayo de 1985 .

Esta vez se sembraron 50 semillas por casilla, con el objeto de aumentar las posibilidades de un resultado mejor. La profundidad de siembra fue de $1-1.5 \mathrm{~cm}$.

\section{Metodologia de Análisis}

Con los resultados de sobrevivencia (considerando un $80 \%$ de germinación obtenido en laboratorio) de cada tratamiento y subparcela (grupo de 4 casillas de siembra) se aplicó un análisis de varianza para un diseño de bloques al azar. Los rangos de significación estadística ( $95 \%$ de probabilidad) se establecieron mediante el test de Duncan (STEEL and TORRIE. 1960).

\section{RESULTADOS}

\section{Ensayos establecidos en 1985}

En el período Junio 1985 a Mayo 1986, se hicieron cuatro mediciones, determinándose una fuerte mortalidad entre las tres primeras observaciones. Para fines prácticos se considera de interés el número de plantas que logró sobrevivir todo el primer período seco, no importando la supervivencia en las observaciones dentro del periodo menor.

El conteo de supervivencia y medición de las plántulas. un año después del establecimiento, en Mayo 1986, arrojó los resultados que se indican en el Cuadro 3. Al aplicar el test de Duncan se determinó que existen diferencias significativas en el ensayo costero entre el tratamiento "b" (mulch - riego) comparado con los tratamientos "d" y "e" (sin riego).

\section{CUADRO 3}

\section{RESULTADOS DE SIEMBRA DIRECTA UN AÑO DESPUES DEL ESTABLECIMIENTO}

\begin{tabular}{|c|c|c|c|c|}
\hline Tratamiento & Lugar de & Ensayo & $\begin{array}{c}N^{\circ} \text { plantas vivas } \\
\text { (suma de } 6 \\
\text { repeticiones) } \\
\text { (2) }\end{array}$ & $\begin{array}{c}\text { Altura media } \\
\text { de la } \\
\text { Planta } \\
\text { (cm) }\end{array}$ \\
\hline $\begin{array}{l}\text { a) E.S. - sombra - riego (1) } \\
\text { b) E.S. - mulch-sombra-riego } \\
\text { c) E.S. - riego } \\
\text { d) E.S. } \\
\text { e) Testigo }\end{array}$ & $\begin{array}{l}\text { Costa } \\
\text { Interior } \\
\text { Costa } \\
\text { !nterior } \\
\text { Costa } \\
\text { Interior } \\
\text { Costa } \\
\text { Interior } \\
\text { Costa } \\
\text { Interior }\end{array}$ & $\begin{array}{l}\text { ("La Totora") } \\
\text { ("La Jarilla") } \\
\text { ("La Totora") } \\
\text { ("La Jarilla") } \\
\text { ("La Totora") } \\
\text { ("La Jarilla") } \\
\text { ("La Totora") } \\
\text { ("La Jarilla") } \\
\text { ("La Totora") } \\
\text { ("La Jarilla") }\end{array}$ & $\begin{aligned} 7 & (1.4) \\
6 & (1.2) \\
13 & (2.7) \\
8 & (1.7) \\
8 & (1.7) \\
2 & (0.4) \\
0 & (0, \\
4 & (0.8) \\
1 & (0.2) \\
0 & (0,\end{aligned}$ & $\begin{array}{l}2.0 \\
2.7 \\
4.4 \\
3.6 \\
2.9 \\
2.2 \\
2.5 \\
2.5 \\
2.5 \\
\therefore\end{array}$ \\
\hline TO T A L & $\begin{array}{l}\text { Costa } \\
\text { Interior }\end{array}$ & $\begin{array}{l}\text { ("La Totora") } \\
\text { ("La Jarilla") }\end{array}$ & $\begin{array}{l}29 \\
20\end{array}$ & \\
\hline
\end{tabular}

NOTA: (1) E.S. = Escarificación del suelo

(2) Se indica entre paréntesis el porcentaje de plantas obtenidas en relación al número de semillas con capacidad germinativa. 


\section{Ensayo establecido en 1986}

A fines de Marzo de 1987 (otoño), casi un año después del establecimiento (9 meses) st efectuó la evaluación. Los resultados se entregan en el Cuadro 4.

\section{CUADRO 4}

\section{RESULTADOS DE SIEMBRA DIRECTA 9 MESES DESPUES DEL ESTABLECIMIENTO EN "LA TOTORA" (COSTA DE VALLENAR)}

\begin{tabular}{|c|c|c|c|c|}
\hline Tratamiento & & $\begin{array}{l}\mathrm{N}^{\circ} \text { total de } \\
\text { plantas vivas } \\
\text { (\%) (1) }\end{array}$ & $\begin{array}{l}\text { Altura media } \\
\text { de la planta } \\
\text { (cm) }\end{array}$ & $\begin{array}{l}\text { \% de casillas } \\
\text { con plantas }\end{array}$ \\
\hline $\begin{array}{l}\text { Preparación intensiva de Suelo: } \\
\text { Riego cada } 3 \text { meses } \\
\text { Riego cada } 2 \text { meses } \\
\text { Riego cada } 1 \text { mes } \\
\text { Absorbente de Agua }\end{array}$ & $\begin{array}{l}\text { (IF,) } \\
\text { (IF) } \\
\text { (IF) } \\
\text { (IH) }\end{array}$ & $\begin{aligned} 84 & (10.5) \\
152 & (19.0) \\
187 & (23.4) \\
95 & (11.9)\end{aligned}$ & $\begin{array}{l}4.4 \\
4.7 \\
5.1 \\
4.4\end{array}$ & $\begin{array}{l}80 \\
80 \\
95 \\
60\end{array}$ \\
\hline $\begin{array}{l}\text { Preparación moderada de Suelo: } \\
\text { Riego cada } 3 \text { meses } \\
\text { Riego cada } 2 \text { meses } \\
\text { Riego cada } 1 \text { mes } \\
\text { Absorbente de Agua }\end{array}$ & $\begin{array}{l}\left(\mathrm{MF}_{1}\right) \\
\left(\mathrm{MF}_{2}\right) \\
\left(\mathrm{MF}_{3}\right) \\
(\mathrm{MH})\end{array}$ & $\begin{array}{r}128(16.0) \\
84(10.5) \\
68(8.5) \\
60(7.5)\end{array}$ & $\begin{array}{l}4.8 \\
4.5 \\
5,3 \\
4.5\end{array}$ & $\begin{array}{l}80 \\
50 \\
90 \\
45\end{array}$ \\
\hline
\end{tabular}

NOTA: (1) Entre paréntesis se entrega el porcentaje de plantas obtenidas en relación al número de semillas con capacidad germinativa.

Tal como en el Cuadro 3, el número de plantas vivas corresponde a la suma de las repeticiones, por ejemplo, en el tratamiento $\mathrm{IF}_{3}$ el número medio de plantas vivas es 37.415 repeticiones) y por casilla de siembra es de 9.3 (4 casillas por cada unidad de muestreo).

Como en el ensayo de 1985, se aplicó un análisis de varianza con las plantas sobrevivien. tes (basado en una germinación del $80 \%$ ), se encontraron diferencias significativas.

\section{DISCUSION}

Las respuestas a la siembra son bajas si se comparan las plantas resultantes con el número de semillas sembradas y potencialmente germinables por casilla. El mejor resultado indica que se obtuvieron alrededor de 9 plantas por casilla (23\% de las semillas germinables) (tratamiento $\mathrm{IF}_{3}=187$ plantas obtenidas en 20 casillas de siembra). Estos resultados corresponden a los esperados, considerando las condiciones ambientales muy difíciles. Observando el número de casillas de siembra con plantas vivas, es importante destacar que en casi todos los tratamientos sobre el $50 \%$ de las casillas muestran una respuesta positiva. Esto es importante para propósitos de forestación, puesto que el establecimiento de una planta por casilla es suficiente para asegurar la repoblación. En todo caso, en experiencias a mayor escala debiera considerarse el uso de casillas más pequeñas por razones de costo $(20 \mathrm{~cm} \times 20 \mathrm{~cm})$.

Se analizan a continuación los principales factores que afectaron los resultados. 


\section{Riego y mulch}

Es imposible obtener germinación y desarrollo de plántulas en la siembra directa sin un riego inicial en el área de estudio. Sorprendentemente la frecuencia de riego cada 1 ó 3 meses no afecta significativamente la supervivencia de las plántulas, pero una frecuencia mayor deberia aumentar el crecimiento inicial.

Es necesario destacar que el riego es efectivo solamente con mulch. Los resultados obtenidos en 1985 y en 1986 (todos los tratamientos con mulch) están influenciados por el efecto de la humedad y temperatura del suelo (GOOR, A. and BARNEY, G.W., 1976). Es importante anotar que una capa de paja más gruesa que $3 \mathrm{~cm}$ puede llevar a una debilidad inicial en la planta.

El tratamiento con polımero absorbente de agua (Hydro-Growth) no incidió en una supervivencia mayor. esto si se compara con tratamientos con igual frecuencia de riego $\left(\mathrm{F}_{1}\right)$. La cantidad del polímero usado por casilla (10 gr) es menor al recomendado por el fabricante (aprox. $25 \mathrm{gr}$ ). Se aplicó una cantidad menor de producto pensando que la Algarrobilla es una especie que requiere muy poca humedad. Es posible que realmente la cantidad de polímero haya sido insuficiente.

\section{Preparación de suelo}

Debido a que se trata de un suelo compactado y de formación incipiente, no es posible practicar siembra directa sin una preparación del suelo. Es muy probable que la influencia de la intensidad de preparación de suelo se manifieste más adelante. Se recomienda, por lo tanto, la preparación más intensiva.

\section{Protección a la siembra}

Es necesario proteger la siembra del ganado caprino y los herbívoros silvestres (ratas y liebres). La protección de cabras se logró con cerco.

En los ensayos de 1985, las ratas causaron una alta mortalidad, pero el daño debido a esta causa no pudo ser evaluado. Se pensó que el cerco de malla alrededor de todo el ensayo sería suficiente protección contra ratas y liebres, lo que no fue así.

El control de roedores se logró mediante cebos venenosos, aumentando notoriamente el número de plantas vivas en todos los tratamientos del experimento de 1986, si se compara con el de 1985 ( $23 \%$ plantas resultantes contra $2,7 \%$ en el tratamiento más favorable).

Cada casilla de siembra se podría haber protegido individualmente mediante una malla fina, para evitar daños de herbívoros.

La protección contra herbívoros es una práctica común en la siembra directa y es de mayor importancia en zonas áridas (MC MINN, 1970. BURNS and REYNOLDS, 1975. GOOR and BARNEY, 1976).

De acuerdo a los resultados de algunos autores (BURNS and HEBB, 1970; MC MINN, 1970; BARNETT, J.P., CAMPELL, T.E. and DOUGHERTY, P., 1974) la profundidad de siembra es un tema de interés de investigar, con el objeto de proteger la semilla de animales pequeños y de la sequía.

El tamaño de la semilla de Algarrobilla, parecida a un garbanzo (6.400 - 7.300 semillas por $\mathrm{Kg}$ ), permite una buena manipulación para la siembra.

\section{Otros factores}

Experiencias en producción de plantas de Algarrobilla han demostrado una mortalidad alta a partir del tercer mes después de la germinación. La causa es una infección por el hongo 
Fusarium oxysporun (DONOSO J., 1985; MONTENEGRO, G., y ARCE, P., 1987). Es posible que este factor se encuentre presente en estos experimentos de siembra, pero no se conoce su magnitud.

Se desconoce el comportamiento de la especie en cuanto a la sobrevivencia futura, pasado el primer período de sequía.

$\mathrm{El}$ año 1986 fue más favorable en lluvias que el anterior, lo que también influye en un mejor resultado. La precipitación en Vallenar en 1985 fue $29,4 \mathrm{~mm}$ y en 1986 fue de $40,0 \mathrm{~mm}$.

\section{CONCLUSIONES}

A pesar de las condiciones adversas del medio ambiente, se obtuvo alguna respuesta al primer año en ensayos de siembra directa con Algarrobilla (Balsamocarpon brevifolium CLOS).

Se recomienda un riego inicial una vez por mes (equivalente a $8 \mathrm{It}$ por casilla de $50 \mathrm{~cm} \mathrm{x}$ $50 \mathrm{~cm}$ ), escarificación del suelo hasta $20 \mathrm{~cm}$ de profundidad y mejoramiento del suelo mediante "mulching". El riego debe mantenerse a través del primer período seco después de la siembra (6 riegos). Es necesaria una protección contra roedores.

La semilla debe ser tratada previamente (escarificación en ácido sulfúrico).

\section{AGRADECIMIENTOS}

Estos ensayos se llevaron a cabo como parte del Proyecto sobre Taninos Vegetales, financiado por el Centro Internacional de Investigaciones para el Desarrollo del Gobierno de Canadá (CIID).

Los Ingenieros Forestales Srs. Patricio Rojas y Manuel Arriagada, junto con el Técnico Sr. Roger Carrasco, participaron en la instalación y/o mantención de los ensayos. El Ingeniero Forestal Sr. Sergio Aguirre colaboró en la traducción de este informe al idioma inglés. Se agradece la colaboración y apoyo logístico del personal de la Corporación Nacional Forestal (CONAF) en Vallenar.

\section{REFERENCIAS BIBLIOGRAFICAS}

1. ALMEYDA, E., y SAEZ, S. 1958. Recopilación de datos climáticos de Chile. Santiago. Chile, Ministerio de Agricultura. $195 \mathrm{p}$.

2. ANTONIOLETTI et al. s.f. Características climáticas del Norte Chico. Santiago, Chile. Instituto de Investigaciones de Recursos Naturales. $102 \mathrm{p}$ y anexos.

3. BARNETT, J.P.; CAMPBELL. T.E., y DOUGHERTY, p. 1984. Seedling establishment Artificial methods. Reprinted from Proc, of the Symposium on the loblolly pine ecosystem (West Region). March 20 - 22, 1984. Jackson. M.S. Edit. by Bob L. Karr, J. Baker and T. Monaghan, pp. 109-125.

4. BURNS, R. and HEBB, E. 1972. Site preparation and reforestation of droughtacid sands. USDA Forest Service, Agriculture Handbook $\mathrm{N}^{\circ} 426.60 \mathrm{p}$.

5. BURNS, R. and REYNOLDS, R. 1975 Planting dates for longleaf, slash and sand pine seeds in the South Eastem Sandhills. Effect of temperature and rainfall. USDA Forest Service, Research Paper SE - 143. 17 p.

6. CORPORACION DE FOMENTO DE LA PRODUCCION (CORFO). 1984. Análisis de los ecosistemas de la III Región de Chile. Santiago, Chile. CORFO. 281 p.

7. DI CASTRI, F., y HAJEK, E. 1976. Bioclimatología de Chile. Santiago, Chile. Vicerrectoría Académica de la Universidad Católica de Chile. 128 p.

8. ESCOBAR, S.A. 1946. Contribución al estudio de la Algarrobilla. Tesis. Universidad de Chile. Facultad de Agronomía. Santiago, Chile, 26 p.

9. FUENZALIDA, H. 1967. Climatología. En: Corporación de Fomento de la Producción: Geografía Económica de Chile, Santiago, Chile,, CORFO, Ed. Universitaria, p. 99 - 152.

10. GOOR, F. N., and BARNEY, C.W. 1978 Forest tree planting in arid zones. 2nd ed. New York, the Ronald Press Co. 504 p. 
11. HOVES. F. N. 1953. Vegetable tanning materials, London, Butterworths Scientific Publications. 325 p.

12. MC MINN, J.W. 1970. Optimum depth and seanos for direct seeding slash pine in South florida. USDA Forest Service. Research paper, SE-73. 7 p.

13. MONTENEGRO, G., y ARCE, P. 1987. Producción de plantas de Balsamocarpon brevifolium (Algarrobilla) Informe para INFOR, Instituto Forestal, Santiago, Chile. 14 p.

14. ORTIZ, G. J. 1943, La Algarrobilla, valioso arbusto industrial de la región nonte. Revista Simiente (Chile) 13(1). $36 \cdot 39$.

15. ROBERTS, P.C., y DIAZ. C. 1959 - 60. Grandes Grupos de Suelos de Chile. En: Agricultura Técnica. Chile. Años XIX y XX. pp $7-36$.

16. STEEL. R., and TORRIE. J. 1960. Principles and procedures of statistics with special reference to the biological sciences, New York, Mc Graw Hill, 481 p. 